\title{
O cuidado em saúde mental no CAPS no entendimento dos profissionais
}

\author{
M ental care delivered in psychosocial care centers (CAPS) \\ from the viewpoint of the professionals
}

Fernanda Barreto M ielke ${ }^{1}$ Luciane Prado Kantorski ${ }^{2}$

Vanda Maria da Rosa Jardim ${ }^{2}$

Agnes Olschowsky ${ }^{3}$

$\mathrm{M}$ arlene Silva $\mathrm{M}$ achado ${ }^{4}$

\footnotetext{
1 Programa de Pós-

Graduação em Enfermagem,

Escola deEnfermagem,

UFRGS. RuaCel. Fernando

M achado 984/1704, Centro.

90010-320. Porto Alegre

RS. fbmielke@gmail.com

${ }^{2}$ Faculdade deEnfermagem

eObstetrícia, UFPEL.

${ }^{3}$ Escola deEnfermagem,

UFRGS.

${ }^{4}$ CAPS Fragata, Pelotas

(RS).
}

Abstract This article deals with a subproject of the Project for Evaluation of the CAPS psychosocial care centers (CAPS) in the southern region of Brazil, whose purpose was to learn how the professionals of a substitute service feel about of the mental care delivered there. The study was conducted in a CAPS II using a qualitative approach. Data were collected in interviews. The data were classified into three thematic groups and analyzed according to the available literature. In this article we will approach the understanding of the professionals about the care services offered in the CAPS. The results show that for these professionals care involves more than the biological aspects, including the family and society. We perceive that the team is extending its perception of mental health, when considering psychosocial rehabilitation the most important aspect.

Key words Nursing, M ental Health, Rehabilitation
Resumo Trata-sedeum subprojeto da pesquisa Avaliação dos CAPS da região sul do Brasil, tendo como objetivo conhecer 0 entendimento dos profissionais de um serviço substitutivo sobre o cuidado em saúde mental prestado neste espaço. Estudo com abordagem qualitativa, realizado em um CAPS II. A coleta dos dados ocorreu através de entrevistas. Os dados foram classificados em três temáticas, analisados segundo a literatura disponível. Neste artigo, abordaremosa temática quetrata do entendimento dos profissionais do serviço sobre o cuidado que é prestado neste local. Os resultados mostram que o cuidado para estes profissionaisabrangeaspectos quevão além do biológico, incluindo também a família e a sociedade. Percebemosquea equipe do serviço está ampliando seu olhar sobre a saúde mental, quando compreende a reabilitação psicossocial como o centro do cuidado.

Palavras-chave Enfermagem, Saúde mental, Reabilitação 


\section{Introdução}

Os serviços substitutivos ao modelo hospitalocêntrico, o mais antigo modelo de cuidado ao portador de sofrimento psíquico, surgem na intenção de que este sujeito doente seja visto a partir de um outro paradigma, o da reabilitação psicossocial, entendida como uma ação ampliada, que considera a vida em seus diferentes âmbitos: pessoal, social ou familiar ${ }^{1}$, objetivando, assim, a reinserção deste sujeito na sociedade.

As relações sociais que se desenvolvem no interior do hospital psiquiátrico são dominantes, evidenciando a hierarquia, subordinação, exclusão, expropriação do saber e a divisão do trabalho e dos saberes em especialidades. A implantação do modelo psicossocial tem o objetivo de reinserir o portador de transtorno psíquico em suas atividades diárias, tornando possível a interação com a família e comunidade em geral ${ }^{2}$.

Desta forma, sob este novo olhar, surgem os serviços substitutivos ao hospital psiquiátrico, que seconstituem em uma rede de atenção à saúde mental. Esta redeéconstituída tanto pela atenção básica em saúde, como as unidades básicas desaúde, quanto pelos serviços especial izados, incluindo ambulatórios de saúde mental, os Centros de Atenção Psicossocial (CAPS), hospital-dia, serviços deurgência eemergência psiquiátricas, leito ou unidadeem hospital geral eserviços residenciais terapêuticos ${ }^{3}$.

Os Centros de Atenção Psicossociais (CAPS) são serviços de atenção diária em saúde mental, de caráter substitutivo ao hospital psiquiátrico. Têm a responsabilidade de atender pessoas com transtornos mentais severos e persistentes, trabal hando sob a lógica da territorialidade. Estes serviços são regulamentados pela portaria ministerial GM no 336, de 19 defevereiro de $2002^{4}$.

0 CAPS trabalha com equipe multiprofissional e as atividades desenvolvidas neste espaço são bastante diversificadas, oferecendo atendimentos em grupos e individuais, oficinas terapêuticas e de criação, atividades físicas, atividades lúdicas, arteterapia, além da medicação, que antes era considerada a principal forma de tratamento. N este serviço, a família é considerada como parte fundamental do tratamento, tendo atendimento específico (grupal ou individual) e livre acesso ao serviço, sempre que se fizer necessário.

Considerando a caminhada ainda breve dos serviços substitutivos e 0 fato dos CAPS serem considerados como ordenadores da rede pelo $\mathrm{M} \mathrm{i-}$ nistério da Saúde, surge uma preocupação com relação ao risco de manicomialização destes serviços, visto que muitos de seus profissionais traba-
Iham ou trabalharam em hospitais psiquiátricos, havendo assim, necessidade de educação em serviço, maiores investimentos em estudos e pesquisas na área ${ }^{5}$.

Com a mudança na assistência à saúde mental proposta pela Reforma Psiquiátrica, a qual implicou a reorganização das práticas assistenciais, a avaliação nos serviços da área vem crescendo e configurando-se como uma necessidade nos últimos anos. Assim, a construção de processos avaliativos está sendo desenvolvida, considerando os atores os diversos usuários, familiares, profissionais, entre outros.

A partir do Projeto Multicêntrico de Avaliação de Qualidade de Serviços de Saúde M ental em Municípios Brasileiros, foi estudada a questão da satisfação, em especial, de usuários e familiares como fatores de avaliação da qualidade dos serviços de saúde mental ${ }^{6}$.

Porém, estudos de avaliação deserviços de saúde mental a partir dos profissionais são pouco desenvolvidos, fazendo-se necessários.

Escalas para avaliar o grau de sobrecarga e de sati sfação da equipes dos serviços de saúde mental foram reformuladas e adaptadas das originais canadenses para o contexto brasileiro para contemplar este grupo de atores destes serviços e também para servir de estímulo aos estudos com estes sujeitos, com enfoque avaliativo ${ }^{7}$.

Desta forma, entendemos que a avaliação pode ser compreendida eutilizada como um instrumento potencial para a efetivação das práticas psicossociais, no contexto da mudança paradigmática em saúde mental. E é de grande importância que as pesquisas avaliativas sejam realizadas a partir do cotidiano do serviço, considerando a complexidade do objeto da saúde mental ${ }^{8}$.

Assim, estetrabalho busca conhecer o entendimento dos profissionais de um CAPS II da região Sul do Brasil sobre o cuidado que é prestado neste serviço ao portador de transtorno psíquico.

\section{M etodologia}

Este trabalho é parte da pesquisa intitulada Avaliação dos Centros de Atenção Psicossocial da Região Sul do Brasil - CAPSUL, tendo obtido aprovação do Comitê de Ética em Pesquisa da Faculdade de M edicina da Universidade Federal de Pelotas, ofício no 068/06, em 08 de agosto de 2006.

0 presente estudo originalmente foi composto deduas etapas: quantitativa e qual itativa. Aqui nos deteremos na etapa qual itativa, de caráter descritivo e exploratório. 
0 estudo foi realizado em um CAPS II de um município do Rio Grande do Sul. Os sujeitos do estudo foram dezoito profissionais de um total de 22 , sendo que três estavam em período de férias e um não aceitou participar do estudo. Estenúmero de profissionais representa cerca de $82 \%$ da equipe do CAPS.

Os dados foram coletados através de uma entrevista semi-estruturada, contendo três questões norteadoras. Estas foram gravadas e transcritas na íntegra. A coleta ocorreu em dezembro de 2006.

Todos os participantes assinaram o termo de consentimento livre e esclarecido, que continha explicações sobre 0 estudo e igualmente garantia 0 anonimato e a confidencialidade dos dados coletados. Os entrevistados foram identificados pela letra T (trabalhador) acompanhada do número da entrevista.

Os aspectos éticos foram devidamente respeitados, mantendo a observância do Código de Ética dos Profissionais de Enfermagem de 2001 e da Resolução no 196/1996 do Conselho Nacional de Saúde do M inistério da Saúde.

Os dados obtidos foram organizados em temáticas ${ }^{9}$ e analisados conforme a literatura disponível e os objetivos do trabalho.

\section{O campo de estudo}

Trata-se de um Centro de Atenção Psicossocial CAPSII, situado em um município da região Sul do Brasil.

O serviço em estudo iniciou o atendimento em saúde mental em 1992, junto a uma Unidade Básica de Saúde, mas com equipe própria, estruturada pela Secretaria M unicipal de Saúde do município.

Em 2002, em um prédio próprio, o serviço passou a desenvolver suas atividades em regime de atenção diária, funcionando segundo as normas do CAPS. 0 cadastramento no M inistério da Saúde ocorreu em final deste ano.

A equipe que compõe o serviço é formada por duas enfermeiras, três auxiliares de enfermagem, três psicólogas, uma médica psiquiatra, um médico clínico geral, uma assistente social, uma artista visual, uma artesã, dois professores de educação física, uma professora de música, dois merendeiros, uma profissional de serviços gerais, três burocratas, totalizando 22 profissionais de níveis mé dio esuperior, queatendem das 8:00 às 12:00 edas 13:00 às 17:30 horas. 0 almoço é servido no intervalo das 12:00 às 13:00 horas para os usuários intensivos, acompanhados pelos profissionais.
0 serviço atende atualmente cerca de 600 usuários por mês, distribuídos nas três modalidades de atendimento previstas na legislação: intensivo, semi-intensivo enão-intensivo.

O CAPS possui coordenação local, que é eleita pelos usuários, familiares e profissionais do serviço. Atualmente uma psicóloga é coordenadora há um ano e três meses.

No serviço são desenvolvidas atividades como: triagem, atendimentos individuais e em grupos, oficinas terapêuticas e de criação, atendimento à família, visitas domiciliares, atividade físicas, assembléia de usuários ereunião de equipe, ocorrendo, as duas últimas, semanalmente.

O CAPS em questão é ainda um espaço de ensino. Recebe acadêmicos, principalmente, de enfermagem, que têm a oportunidade de conhecer a proposta do serviço e colaborar no cuidado, buscando deste modo qualificar as intervenções dessa equipe. A presença dos acadêmicos é bem aceita pelos profissionais e, especialmente, pelos usuários e seus familiares.

\section{Resultados ediscussão}

Dos dados coletados nas entrevistas surgiram três temáticas: 0 entendimento dos profissionais sobre cuidado em saúde mental, a caracterização do cuidado em saúde mental prestado pelos profissionais do CAPS e as dificuldades e deficiências encontradas no serviço. Neste artigo, abordaremos a temática do entendimento dos profissionais sobre cuidado em saúde mental. 0 questionamento realizado nas entrevistas foi: "Q ual o seu entendimento acerca do cuidado em saúde mental?".

\section{0 entendimento dos profissionais} sobre cuidado em saúde mental

Os profissionais do serviço em estudo consideram que o cuidado em saúde mental segue os princípios da integralidade, assistindo o usuário em todas as áreas do ser humano: biopsicossocial eespiritual, não fragmentando o cuidado. Apontam também como sendo um cuidado humanizado, existindo vínculos entre equipe e usuário e havendo a responsabilização de ambos pelo cuidado. Essas características são apontadas nas falas que seguem.

U m cuidado abrangente, não só da saúde mental. T4

Parece que quando a gente pega uma área é só aquela área, não, para mim é abrangente. T 7 
Todos tratados com carinho, com atenção. T10

0 entendimento da saúde mental é só saber ser humano. T12

Eu acho que a genteé um todo emocional que na verdade o nome pra mim é muito mais esse do que mental, e até espiritual. T14

0 cuidado ao ser humano é um ato complexo, que exige do cuidador conhecimento, empatia e sensibilidade. 0 cuidado também envolve tarefas como tocar, sentir, escutar e auxiliar o outro nas atividades em que ele apresenta dificuldade $\mathrm{e}^{10}$. Os profissionais do serviço durante as atividades buscam abrir espaços para conversas individuais ou mesmo com o grupo no intuito de aliviar ansiedades, medos e discutir situações específicas que são vivenciadas pelos usuários.

A humanização também é compreendida como estratégia de interferência no processo de produção da saúde, buscando o alcance da qualificação da atenção e da gestão em saúde no Sistema Ú nico de Saúde. É garantir o acesso universal, integral e imparcial com acolhimento eresolutividade, superando a compartimentalização da assistência em saú$\mathrm{de}^{11}$. Isto significa oferecer um atendimento de qualidade contando com as novas tecnologias como 0 acolhimento, melhorias do ambiente de cuidado e também condições de trabalho aos profissionais.

Para haver o cuidado humanizado, é necessário ocorrer empatia, afetividade, envolvimento e aproximação entre cuidador e aquele que é cuidado com finalidade terapêutica, não se limitando apenas às características das técnicas ${ }^{12}$. Durante as entrevistas, muitos profissionais relataram o forte vínculo que é criado entre eles e os usuários. Este vínculo é visto pela equipe como facilitador no tratamento, havendo fidelidade entre ambas as partes. Assim, o cuidado em saúde mental ganha materialidade na atitude dos profissionais do CAPS na sua relação com os usuários, tendo a integralidade como foco de intervenção, ou seja, éum local de encontro, diálogo entre pessoas com necessidades, desejos, histórias e conhecimentos específicos.

0 cuidado para esses profissionais envolvetambém a família do usuário, que é entendida como parte fundamental para a evolução satisfatória do usuário no paradigma psicossocial de atenção à saúde mental.

Nas falas seguintes, percebemos a tendência de inclusão da família no tratamento ao portador de transtorno psíquico.

Incluindo a família sempre, de preferência. T4 T17

Em primeiro lugar os familiares que ajudassem.

Sempre incluindo a família, éum trabalho sempre muito com a família. T18
Os profissionais apontam que há famílias que buscam auxílio no serviço, que participam das atividades propostas, como os grupos de familiares, e outras que nem procuram saber da situação de seu familiar no serviço.

Porque os familiares não estão nem aí. T1

A pesar de muitas vezes ser difícil porque eles não querem ser incluídos. T4

A família também está desgastada. T15

Aqueles que os familiares não ajudam. T17

A gente tem que buscar essa família e tem tido assim grandes resultados. T18

Entendemos que a participação da família no tratamento é importante para os profissionais do CAPS, como nos mostram as fal as, nas quais apontam que há responsabilidade dos familiares nessa parceria para o tratamento.

Atualmente, é consenso de que as famílias, quando recebem apoio e orientação adequados, têm condições de compartilhar seus problemas e tornam-se aliadas na desinstitucionalização e na reabilitação social do usuário ${ }^{13}$. É necessário oferecer aten ção e apoio a estas famílias, pois a reinserção do usuário na comunidade e a retomada de suas atividades diárias se tornam mais fáceis e rápidas quando os familiares acreditam que a meIhora na condição de saúde do usuário é possível.

Por outro lado, entendemos que a ausência de alguns familiares nas atividades do serviço está relacionada às dificuldades em assumir a responsabilidade que Ihe cabe no tratamento, justificada pelo sentimento de sobrecarga da mesma com relação ao usuário.

A sobrecarga da família do usuário portador de transtorno mental referese às conseqüências que afetam o cotidiano desta família. Os gastos financeiros, a desestruturação da vida familiar, social eprofissional, tarefas extras, convivência com comportamentos impróprios são situações com as quais a família acaba aprendendo a lidar, mas que causam um grande desgaste físico, mental e emocional ${ }^{14}$.

O serviço deve buscar outras formas de alcançar essa família, pois a sensação de responsabilidade, associada à vivência do sofrimento psíquico, é desgastante e o apoio no tratamento e parceria com a equipe é sempre necessário.

A preocupação com a reabilitação psicossocial do usuário também foi entendida como parte do cuidado em saúde mental.

A saúde mental é importante pro usuário, pois ajuda-0 a voltar ao ambiente social. T3

É importante se pensar muito na reabilitação social. A través dela se vai conseguir incluir de novo o paciente psiquiátrico na sociedade. T6 
Acho que isso é um processo muito legal, assim, de focalizar na reabilitação, que não só vê o sintoma, aquela coisa, e de ver o foco sadio dessepaciente. T15

Já vi pessoas entrarem aqui e saírem boas. T16

A reabilitação psicossocial, no contexto da re forma psiquiátrica, define-se como um saber fazer, considerando o transtorno psíquico como um dado da história de vida do usuário, ou seja, um indivíduo que vive em um território, que mantém relações sociais e afetivas, fazendo parte de determinada família e que apresenta um transtorno mental que tem diferentes repercussões em sua vida ${ }^{15}$. 0 ol har ao doente mental é ampliado e este passa a ser visto como cidadão, com direitos e deveres, e co-responsável por seu tratamento e por suas condições de vida.

A reabilitação também é entendida como um conjunto de ações que visam a aumentar as habilidades da pessoa, diminuindo o dano causado pelo transtorno mental, e envolve todos aqueles que fazem parte do processo de saúde- doença, ou seja, usuários, familiares, profissionais e comunidade em geral ${ }^{16}$. Os serviços substitutivos, tais como os CAPS, possuem esse caráter reabilitador por serem serviços territorializados, utilizando espaços na própria comunidade, fazendo com que o usuário, portador de transtorno mental, retome sua posição frente à sociedade.

Nas entrevistas, os profissionais apontam que um dos objetivos de seu trabalho éfazer com que a sociedadetambém participedo processo de reabilitação psicossocial do usuário, para que a inclusão deste aconteça de forma natural. Isto significa a mudança do olhar da sociedade para a pessoa doente: de incapaz para capaz, de louco para doente, de inútil para cidadão. É um processo que envolve construção de autonomia, liberdade e cidadania.

Tem também o negócio da sociedade, quea gente vai conseguindo essa inclusão social. T6

O rientar as pessoas sobre viver bem e sobre a responsabilidade que cada um tem sobre a própria saúde, como um todo. T9

Q ue as pessoas possam ver aquela pessoa assim, com tanto problema psíquico, dando o máximo de si, sendo feliz. T13

A reabilitação é entendida como o resgate de um conceito mais positivo sobre a saúde mental, na qual a pessoa évista como capaz deagir, decidir, opinar, sofrer, al egrar-se, enfim, confrontar-se com o estigma de louco incapaz, concepção que o desvaloriza enquanto cidadão ${ }^{17}$.

Uma das possíveis formas de incluir socialmenteaqueles que sofrem com transtorno mental é por meio de atividades artísticas e artesanais realizadas através de oficinas terapêuticas. As oficinas terapêuticas são descritas pela portaria SN AS no 189/1991 como "atividades grupais de socialização, expressão einserção social" ${ }^{18}$. A dança, a música, a arte, 0 teatro, a marcenaria, entre outros são atividades desenvolvidas em grupo, oportunizando ao usuário a inter-relação com os demais participantes.

As falas que seguem trazem esse aspecto do cuidado ser visto como potencializador para a inserção social.

Possibilita assim a pessoa atingir o seu potencial, desenvolver um potencial que mesmo com a doença ela écapaz. T5

Explorar nele os potenciais que existem, as riquezas que existem no interior dele. T13

Botar potencial na pessoa, mostrar que tu acreditasnela. T15

As oficinas, na proposta da reforma psiquiátrica, são entendidas como impulsionadores da construção de espaços sociais onde o usuário possa reconquistar ou conquistar seu cotidiano ${ }^{19}$.

A oficina terapêutica faz parte do processo reabilitador e, desta forma, se mostra como experiência positiva, atuando no campo da cidadania. É uma maneira também de incentivar a preparação para o mercado de trabalho, fazendo com que 0 usuário retome suas atividades e descubra novas habilidades, na busca por sua independência.

\section{Consideraçõesfinais}

A proposta de cuidado ao portador de transtorno mental no interior dos CAPS é baseada em ações que visam a sua reabilitação psicossocial, na busca da autonomia e da cidadania destas pessoas.

o modelo psicossocial de cuidado ao indivíduo em sofrimento psíquico, alicerçado nos pressupostos da Reforma Psiquiátrica, um movimento de transformações das práticas, saberes, valores culturais e sociais em saúde mental ${ }^{20}$, está se de senvolvendo nestes espaços substitutivos de atenção. 0 objetivo maior deste movimento é a reinserção do sujeito com transtorno mental ao seu território, à sua família e comunidade, oferecendo-Ihe as condições necessárias, tais como cuidado no território, atendimento à família, atividades na comunidade, entre outros. Com este estudo, foi possível perceber que esta mudança de paradigma vem ocorrendo aos poucos, mantendo como horizonte este objetivo de reinserção social.

Os profissionais do serviço em estudo entendem o cuidado em saúde mental como uma ação abrangente, quevai além do cuidado específico com a saúde mental, que envolve a família e a socieda- 
de, quando compreende a reabilitação psicossocial como o centro do cuidado.

A transformação das formas de cuidado em saúde mental mostra-se viável efavorece a efetivação da proposta da reforma psiquiátrica, na qual o usuário recebe um atendimento que respeita sua cidadania e autonomia.

\section{Referências}

1. Saraceno B. Libertando identidades: da reabilitação psicossocial à cidadania possível. 2 a ed. Rio de Janeiro: Te Cora; 2001.

2. Costa-Rosa A. O modo psicossocial: um paradigma das práticas substitutivas ao modo asilar. In: Amarante $P$, organizador. Ensaios: subjetividade, saúde mental, sociedade. Rio de Janeiro: Fiocruz; 2000. p.141-168.

3. Brasil. M inistério da Saúde. Guia de saúde mental Porto Alegre; Ministério da Saúde; 2001.

4. Brasil. Portaria GM $n^{\circ} 336$, de 19 de fevereiro de 2002. Define e estabelece diretrizes para o funcionamento dos Centros de Atenção Psicossocial. Diário O ficial da União 2002; 20 fev.

5. Onocko-Campos RT, Furtado JP. Entre a saúde coletiva e saúde mental: um instrumental metodológico para avaliação da rede de Centros de Atenção Psicossocial (CAPS) do Sistema Ú nico de Saúde. Cad Saúde Pública 2006; 22(5):1053-1062.

6. Silva Filho JF, Cavalcanti MT, Cadilhe GR, Vilaça AT, Avancini E, Machado HO, Perecmanis L, Maisonnette MJ, Rocha EC, Carreteiro RO, Mozinho GM P, Pitta AM. Avaliação de qualidade de serviços de saúde mental no M unicípio de Niterói-RJ: a satisfação dos usuários como critério avaliador. J. Bras. Psiquiatr. 1996; 45(7):393-402.

7. Bandeira M, Pitta AM F, M ercier C. Escalas brasileiras de avaliação de satisfação (SATIS-BR) e da sobrecarga (IM PACTO-BR) da equipe técnica em serviços de saúde mental. J. Bras. Psiquiatr. 2000; 49(4):105-115.

8. Wetzel C, Kantorski LP. Avaliação de serviços em saúde mental no contexto da Reforma Psiquiátrica. Texto Contexto Enferm 2004; 13(4):593-598.

9. M inayo MCS. 0 desafio do conhecimento: pesquisa qualitativa em saúde. São Paulo: Hucitec; Rio de Janeiro: Abrasco; 1996.

10. Soares MC, Santana M G, Siqueira H. O cuidado de enfermagem no cotidiano das enfermeiras (os) autônomas (os). À luz de alguns conceitos da teoria humanística de Paterson e Zderard. Texto Contexto Enferm 2000; 9(2):106-117.

\section{Colaboradores}

FB M ielke, LP Kantorski eV M R Jardim participaram da concepção do trabal ho e do artigo, da análise dos dados e redação final; A O Ischowsky e MS Machado participaram da concepção do artigo, análise dos dados e redação final.

11. Brasil. M inistério da Saúde. Saúde da família: panorama, avaliação e desafios. Brasília: M inistério da Saúde; 2005.

12. Baggio MA. O significado de cuidado para profissionais da equipe de enfermagem. Rev Eletrônica de Enfermagem [periódico na Internet] 2006 [acessado 2007 jan 29] 8(1): [cerca de 8 p]. Disponível em: http:// www.fen.ufg.br/revista/revista8_1/original_01.htm

13. Esperidião E. Assistência em saúde mental. A inserção da família na assistência psiquiátrica. Revista Eletrônica de Enfermagem [periódico na Internet] 2001 [acessado 2007 jan 29]; 3(1). Disponível em: http:// www.fen.ufg.br/revista

14. Bandeira M, Barroso SM. Sobrecarga das famílias de pacientes psiquiátricos. J. Bras. Psiquiatr. 2005; 54(1): 34-46.

15. Kantorski LP, Souza J, Willrich JQ, Mielke FB. O cuidado em saúde mental: um olhar a partir de documentos e da observação participante. R Enferm UERJ 2006; 14(3):366-371.

16. Lussi IAO, Pereira MAO, Pereira Júnior A. A proposta de reabilitação psicossocial de Saraceno: um modelo de auto-organização? Rev Latino-am Enfermagem 2006; 14(3):448-456.

17. Olschowsky A. 0 ensino de enfermagem psiquiátrica e saúde mental: análise da pós-graduação "Iato sensu" [tese]. São Paulo (SP): Escola de Enfermagem, USP; 2001.

18. Brasil. Portaria SAS $\mathrm{n} 0189$, de 19 de novembro de 1991. Altera o financiamento das ações de saúde mental. Diário Oficial da União 1991; 11 dez.

19. Rauter C. Oficinas para quê? U ma proposta ético-estética-política para oficinas terapêuticas. In: Amarante P, organizador. Ensaios: subjetividade, saúde mental, sociedade. Rio de Janeiro: Fiocruz; 2000. p. 267-277.

20. Brasil. Ministério da Saúde. Reforma psiquiátrica e política de saúde mental no Brasil. Brasília: Ministério da Saúde; 2005.

Artigo apresentado em 04/08/2007

Aprovado em 29/10/2007 